\title{
THE COSMOLOGICAL CONSTANT OF ONE-DIMENSIONAL MATTER COUPLED QUANTUM GRAVITY IS QUANTISED
}

\author{
JAN GOVAERTS \\ Stellenbosch Institute for Advanced Study (STIAS) \\ Private Bag XI, 7602 Stellenbosch, Republic of South Africa \\ http://www.stias.ac.za \\ Institute of Nuclear Physics, Catholic University of Louvain \\ 2, Chemin du Cyclotron, B-1348 Louvain-la-Neuve, Belgium* \\ E-mail: govaerts@fynu.ucl.ac.be
}

\begin{abstract}
Coupling any interacting quantum mechanical system to gravity in one (time) dimension requires the cosmological constant to belong to the matter energy spectrum and thus to be quantised, even though the gravity sector is free of any quantum dynamics. Furthermore, physical states are also confined to the subspace of the matter quantum states for which the energy coincides with the value of the cosmological constant. These general facts are illustrated through some simple examples. The physical projector quantisation approach readily leads to the correct representation of such systems, whereas other approaches relying on gauge fixing methods are often plagued by Gribov problems in which case the quantisation rule is not properly recovered. Whether such a quantisation of the cosmological constant as well as the other ensuing consequences in terms of physical states extend to higher dimensional matter-gravity coupled quantum systems is clearly a fascinating open issue.
\end{abstract}

\author{
Dedicated to John R. Klauder \\ on the occasion of his $70^{\text {th }}$ birthday
}

\section{Introduction}

A basic understanding of the gravitational interaction both in the quantum realm as well as in its relation to the other fundamental interactions and matter, as witnessed for instance amongst other equally relevant open

${ }^{*}$ Permanent address. 
issues by the cosmological constant problem, ${ }^{1}$ is of central importance in all present attempts at a fundamental unification. Being the example par excellence of a system possessing a local gauge symmetry, popular methods for the quantisation of such constrained systems have been brought to bear on the quantisation problem, beginning with pure gravity. On the other hand, given the insight provided by topological quantum field theories of gravity, ${ }^{2,3}$ one is also tempted to flirt with the idea that geometry emerges in fact only through the coupling of gravity to the other fundamental interactions and their matter fields, and that the problems of quantum gravity are to be properly addressed provided only one also includes these other degrees of freedom. Whatever the merits of such a proposal, it certainly does not run counter to all indications motivated by M-theory in this respect.

Given this perspective in the back of one's mind, the aims of the present note are far more modest by addressing these issues for one-dimensional gravity only, leading nonetheless to results that hint possibly at such a connection. Even though gravity is totally free of any classical or quantum degree of freedom in one dimension, we shall find that when coupled to whatever quantum matter system, the cosmological constant must take a quantised value which belongs to the matter energy spectrum, and that the physical states are restricted to belong to the subspace of the matter quantum states for which the energy coincides with the cosmological constant value. Furthermore, in order to assess the merits of different approaches to the quantisation of gauge invariant systems when applied to theories of gravity, three such methods are considered explicitly. It will be shown that Faddeev's reduced phase space approach ${ }^{4,5}$ is unable to properly represent the genuine quantum dynamics of reparametrisation invariant systems, due to Gribov problems. ${ }^{6,5,7,8}$ Likewise, the general BRST quantisation methods ${ }^{10,5}$ are to be trusted only for specific gauge fixing choices which are free of any Gribov problem, while otherwise the correct quantum dynamics is not recovered either. ${ }^{7,8}$ In contradistinction, the physical projector approach of much more recent conception, ${ }^{11,12}$ with in particular its avoidance of any gauge fixing hence also of any Gribov problem, ${ }^{13}$ is shown to be perfectly capable to correctly represent the actual dynamics of one-dimensional matter coupled quantum gravity, including the quantised value of the cosmological constant. Obviously, such conclusions raise the all too intriguing issue of the extension of these results to matter coupled theories of quantum gravity in higher spacetime dimensions. In this respect, the physical projector approach should also prove to be a tool of great efficacy. ${ }^{14}$ 
As a matter of fact, this note grew out from a simple example ${ }^{12}$ of a Gribov problem arising within Faddeev's reduced phase space formulation, to which the physical projector is simply oblivious. This simple example turns out to belong to the general class of systems in which any interacting quantum mechanical matter system is coupled to one-dimensional gravity. Even though the conclusion that in all such models the cosmological constant is to be quantised, is totally general and applies whatever the nature of the interacting quantum matter sector, in the present note we shall only consider a matter sector described by cartesian degrees of freedom. Nonetheless, as the reader will readily realise, the analysis to be presented easily extends to more general cases, such as for instance quantum mechanical nonlinear sigma models of which the degrees of freedom take values in some curved and/or compact manifold.

The sole mention in the literature known to this author of a quantised cosmological constant in the manner discussed here ${ }^{\mathrm{a}}$ is that of Ref. 15, in which the interacting quantum mechanical matter sector is the nonrelativistic hydrogen atom, again a specific example of the general construction to be discussed presently.

The note is organized as follows. In Sec. 2, general quantum mechanical systems, as yet uncoupled to gravity, are considered. In Sec. 3, these systems are coupled to one-dimensional gravity and then quantised following Dirac's general approach, ${ }^{18,5}$ in order to identify their physical sector through the physical projector. ${ }^{11,12}$ It is here that the requirement of a quantised cosmological constant arises explicitly. Section 4 then addresses the same issues, this time from Faddeev's reduced phase space approach, to conclude that this method of fixing the reparametrisation gauge freedom is ill-fated in these systems. In Sec. 5, the Hamiltonian BSRT methods are also brought to bear on the same systems, to conclude once again ${ }^{7,8}$ that even though these methods lead, by construction, to gauge invariant quantities, it is only for an admissible gauge fixing procedure that the correct physical content is properly identified. Section 6 briefly presents some concluding remarks.

\section{The Uncoupled Quantum Matter System}

Quite generally, let us consider an arbitrary classical system for which the Hamiltonian formulation is defined by canonical phase space degrees of free-

a The possibility of a quantised cosmological constant is also mentioned for instance in the papers given in Refs. 16 and 17 based on different considerations. 
dom $\left(q^{n}(t), p_{n}(t)\right)(n=1,2, \cdots, N)$, with the canonical bracket structure $\left\{q^{n}(t), p_{m}(t)\right\}=\delta_{n, m}$, and which possesses a time evolution generated by some Hamiltonian function $H\left(q^{n}, p_{n}\right)$. The dynamics of such a system also follows from the variational principle applied to the first-order phase space Hamiltonian action

$$
S\left[q^{n}, p_{n}\right]=\int d t\left[\dot{q}^{n} p_{n}-H\left(q^{n}, p_{n}\right)\right] .
$$

As indicated previously, we shall assume that the coordinates $q^{n}(t)$ define a cartesian parametrisation of the configuration space of the system, with $q^{n}(t)$ and $p_{n}(t)$ thus taking all possible real values, even though the construction and its conclusions to be presented in Sec. 3 remain valid whatever the choice of matter sector.

In the following, when considering examples, we shall restrict to two specific cases; on the one hand, the single one-dimensional harmonic oscillator of mass $m$ and angular frequency $\omega$, and on the other hand, the $N$-dimensional euclidean spherical harmonic oscillator with the same parameters.

Within this general framework, the classical equations of motion are

$$
\dot{q}^{n}=\frac{\partial H}{\partial p_{n}} \quad, \quad \dot{p}_{n}=-\frac{\partial H}{\partial q^{n}} .
$$

For instance, given initial values $q_{i}^{n}=q^{n}\left(t_{i}\right)$ and $p_{n, i}=p_{n}\left(t_{i}\right)$, the solution to these equations is of the form

$$
q^{n}(t)=Q^{n}\left(t ; q_{i}^{n}, p_{n, i}\right) \quad, \quad p_{n}(t)=P_{n}\left(t ; q_{i}^{n}, p_{n, i}\right),
$$

while the "energy"b of the system is given by the conserved value of the Hamiltonian,

$$
E\left(q_{i}^{n}, p_{n, i}\right)=H\left(q_{i}^{n}, p_{n, i}\right) .
$$

Here, $Q^{n}\left(t ; q_{i}^{n}, p_{n, i}\right)$ and $P_{n}\left(t ; q_{i}^{n}, p_{n, i}\right)$ are specific functions of time, also dependent on the initial values for the phase space degrees of freedom.

As is well known, the Hamiltonian equation of motion for $\dot{q}^{n}$ may be used to reduce the conjugate momenta $p_{n}=p_{n}\left(q^{n}, \dot{q}^{n}\right)$ and obtain the Lagrangian variational principle for the same dynamics,

$S\left[q^{n}\right]=\int d t L\left(q^{n}, \dot{q}^{n}\right) \quad, \quad L\left(q^{n}, \dot{q}^{n}\right)=\dot{q}^{n} p_{n}\left(q^{n}, \dot{q}^{n}\right)-H\left(q^{n}, p_{n}\left(q^{n}, \dot{q}^{n}\right)\right)$,

${ }^{\mathrm{b}}$ Indeed, this quantity measures the actual energy of the system only when the time evolution parameter $t$ coincides with the physical time. 
as well as the Euler-Lagrange equations of motion

$$
\frac{d}{d t} \frac{\partial L}{\partial \dot{q}^{n}}-\frac{\partial L}{\partial q^{n}}=0 .
$$

This reduction is especially simple when the conjugate momenta dynamics separates through an Hamiltonian of the form $H=\sum_{n} p_{n}^{2} /\left(2 m_{n}\right)+V\left(q^{n}\right)$. Examples considered further on belong to this latter characterization.

Within this Lagrangian formulation, a choice of boundary values which is often more convenient than the one above is such that the configuration space values $q^{n}(t)$ are specified both at some initial time $t=t_{i}$ as well as at some final time $t=t_{f}, q_{i, f}^{n}=q^{n}\left(t_{i, f}\right)$. Nonetheless, knowledge of the solutions $Q^{n}\left(t ; q_{i}^{n}, p_{n, i}\right)$ and $P_{n}\left(t ; q_{i}^{n}, p_{n, i}\right)$ enables one in principle to construct the solutions to the Euler-Lagrange equations of motion for this alternative choice of boundary conditions.

The path towards quantisation to be taken in this note is that of the abstract canonical quantisation of the above Hamiltonian formulation. Hence, the quantum system is represented through a linear representation space $\mid \psi>$ of the abstract Heisenberg algebra $\left[\hat{q}^{n}\left(t_{0}\right), \hat{p}_{m}\left(t_{0}\right)\right]=i \hbar \delta_{n, m}$, equipped with an hermitean inner product $<. \mid$. $>$ such that all these operators $\hat{q}^{n}\left(t_{0}\right)$ and $\hat{p}_{n}\left(t_{0}\right)$ be - in the best of cases — self-adjoint. ${ }^{c}$ Finally, time evolution is generated by Schrödinger's equation

$$
i \hbar \frac{d}{d t}|\psi, t>=\hat{H}| \psi, t>
$$

where $\hat{H}=\hat{H}\left(\hat{q}^{n}\left(t_{0}\right), \hat{p}_{n}\left(t_{0}\right)\right)$ is a quantum Hamiltonian operator in correspondence with the classical one, $H\left(q^{n}, p_{n}\right)$, and defined in such a manner that it be self-adjoint as well.

For physical consistency, we shall assume that the $\hat{H}$-spectrum is bounded below. Otherwise, just for the sake of definiteness in our discussion, we shall further assume that this spectrum be discrete, ${ }^{\mathrm{d}}$

$$
\hat{H}\left|E_{n}, \alpha_{n}>=E_{n}\right| E_{n}, \alpha_{n}>\quad, \quad \alpha_{n}=1,2, \cdots, d_{n} \quad, \quad n=0,1,2, \cdots,
$$

with a degeneracy $d_{n} \geq 1$ at each of the energy levels $E_{n}(n=0,1,2, \cdots)$ labelled by $\alpha_{n}=1,2, \cdots, d_{n}$, and with a choice of orthonormalised energy

\footnotetext{
${ }^{\mathrm{c}}$ Canonical quantisation is performed in the Schrödinger picture, so that $t_{0}$ here stands for a reference time at which quantisation is performed for the operator degrees of freedom.

$\mathrm{d}$ The quantisation of the cosmological constant applies whether the matter energy spectrum is discrete or continuous, or a mixture of both.
} 
eigenstates

$$
<E_{n}, \alpha_{n} \mid E_{m}, \alpha_{m}>=\delta_{n, m} \delta_{\alpha_{n}, \alpha_{m}}
$$

which implies

$$
\mathbb{1}=\sum_{n, \alpha_{n}}\left|E_{n}, \alpha_{n}><E_{n}, \alpha_{n}\right| .
$$

Consequently, the Hamiltonian operator possesses the spectral representation

$$
\hat{H}=\sum_{n, \alpha_{n}}\left|E_{n}, \alpha_{n}>E_{n}<E_{n}, \alpha_{n}\right|,
$$

while the time evolution operator $\hat{U}\left(t_{2}, t_{1}\right)=e^{-i / \hbar\left(t_{2}-t_{1}\right) \hat{H}}$ of the quantum system simply reads

$$
\hat{U}\left(t_{2}, t_{1}\right)=\sum_{n, \alpha_{n}}\left|E_{n}, \alpha_{n}>e^{-i / \hbar\left(t_{2}-t_{1}\right) E_{n}}<E_{n}, \alpha_{n}\right| .
$$

Hence, given any initial state

$$
\left|\psi, t=t_{i}>=\sum_{n, \alpha_{n}}\right| E_{n}, \alpha_{n}>\psi_{n, \alpha_{n}} \quad, \quad \psi_{n, \alpha_{n}}=<E_{n}, \alpha_{n} \mid \psi, t=t_{i}>
$$

the general solution to Schrödinger's equation is

$$
\left|\psi, t>=\hat{U}\left(t, t_{i}\right)\right| \psi, t_{i}>=\sum_{n, \alpha_{n}} \mid E_{n}, \alpha_{n}>e^{-i / \hbar E_{n}\left(t-t_{i}\right)} \psi_{n, \alpha_{n}} .
$$

It should be clear how any (of the well known) quantum mechanical system(s) may be brought into correspondence with such a general description, beginning with the ordinary harmonic oscillator in its Fock space representation.

\section{The Gravity Coupled System}

Given any general mechanical system as described in Sec. 2, let us now consider the following first-order action principle,

$$
S\left[q^{n}, p_{n} ; \lambda\right]=\int d t\left[\dot{q}^{n} p_{n}-\lambda\left(H\left(q^{n}, p_{n}\right)-\Lambda\right)\right],
$$

where $\lambda(t)$ is an arbitrary function of $t$ and $\Lambda$ an arbitrary real constant parameter. Clearly, $\lambda(t)$ is a Lagrange multiplier for a constraint on phase space, namely $\phi\left(q^{n}, p_{n}\right)=0$, with

$$
\phi\left(q^{n}, p_{n}\right)=H\left(q^{n}, p_{n}\right)-\Lambda,
$$


while the Hamiltonian equations of motion now read

$$
\dot{q}^{n}=\lambda \frac{\partial H}{\partial p_{n}} \quad, \quad \dot{p}_{n}=-\lambda \frac{\partial H}{\partial q^{n}},
$$

of which the solutions are subjected to the constraint $H\left(q^{n}, p_{n}\right)=\Lambda$. As a matter of fact, (15) defines a constrained system ${ }^{18,5}$ whose phase space coordinates $\left(q^{n}, p_{n}\right)$ are canonical, $\left\{q^{n}, p_{m}\right\}=\delta_{n, m}$, whose time evolution is generated by the total Hamiltonian $H_{T}=\lambda(H-\Lambda)$, and which possesses the gauge invariance degree of freedom under arbitrary local reparametrisations in $t$ generated by the first-class constraint $\phi=H-\Lambda$. The associated first-class Hamiltonian vanishes identically, as befits any reparametrisation invariant system, so that the total Hamiltonian which generates time dependence is indeed "pure gauge", $H_{T}=\lambda \phi$, while the constraint $\phi=0$ that classical solutions must meet expresses their gauge invariance under local time reparametrisations. The fact that (15) does define the coupling of the matter system described by $H\left(q^{n}, p_{n}\right)$ to gravity in a one-dimensional "spacetime" with $\Lambda$ being a cosmological constant may be justified from complementary viewpoints. In particular, $\lambda(t)$ is nothing but a world-line einbein, with $\lambda^{2}(t)$ defining an intrinsic world-line metric. ${ }^{\mathrm{e}}$

First, given initial values $q_{i}^{n}$ and $p_{n, i}$ at $t=t_{i}$ for the phase space degrees of freedom, let us construct the general solutions to the equations of motion (17) whatever the choice for the Lagrange multiplier function $\lambda(t)$. For this purpose, consider the function $\tau(t)$, with $\tau_{i}=t_{i}$, such that

$$
\tau-\tau_{i}=\int_{t_{i}}^{t} d t^{\prime} \lambda\left(t^{\prime}\right) \quad, \quad \tau\left(t_{i}\right)=t_{i}=\tau_{i} .
$$

It then follows that in terms of this variable $\tau$, the equations of motion (17) reduce to those of the uncoupled matter system,

$$
\frac{d q^{n}}{d \tau}=\frac{\partial H}{\partial p_{n}} \quad, \quad \frac{d p_{n}}{d \tau}=-\frac{\partial H}{\partial q^{n}},
$$

while the first-order action (15) reads

$$
S\left[q^{n}, p_{n} ; \lambda\right]=\int d \tau\left[\frac{d q^{n}}{d \tau} p_{n}-\left(H\left(q^{n}, p_{n}\right)-\Lambda\right)\right] .
$$

e Note that the classes of models considered here include the scalar relativistic particle propagating in a spacetime of whatever geometry, the parameter $\Lambda$ being directly related to the particle's squared mass which must indeed belong to the "energy" spectrum of the operator $\hat{P}_{\mu} \hat{P}^{\mu}$. 
Consequently, irrespective of the choice for $\lambda(t)$, the general solution to the equations of motion associated to the initial values $\left(q_{i}^{n}, p_{n, i}\right)$ is of the same form as that for the uncoupled system,

$$
q^{n}(t)=Q^{n}\left(\tau(t) ; q_{i}^{n}, p_{n, i}\right) \quad, \quad p^{n}(t)=P^{n}\left(\tau(t) ; q_{i}^{n}, p_{n, i}\right),
$$

with however the time dependence on $t$ substituted by that on the parameter $\tau(t)$, and the further restriction that the "energy" $E$ of the solution must coincide with the cosmological constant value $\Lambda$,

$$
E\left(q_{i}^{n}, p_{n, i}\right)=H\left(q_{i}^{n}, p_{n, i}\right)=\Lambda .
$$

In other words, irrespective of the choice of world-line (re)parametrisation characterized by the function $\tau(t)$ such that $\tau\left(t_{i}\right)=t_{i}$, and thus associated to the Lagrange multiplier $\lambda(t)=d \tau(t) / d t$, the solution is given by the same functions $Q^{n}\left(\tau ; q_{i}^{n}, p_{n, i}\right)$ and $P_{n}\left(\tau ; q_{i}^{n}, p_{n, i}\right)$ as those of the uncoupled system, with however the restriction that the cosmological constant $\Lambda$ must belong to the $H$-spectrum and that the energy $E$ of the solution must coincide with $\Lambda$. The dependence of the general solution to the equations of motion on an arbitrary function $\tau(t)$ of time is the manifest hallmark of a system which is gauge invariant under local reparametrisations in one dimension, namely in the time parameter. The physical content of such systems is thus that which is totally independent of the world-line parametrisation, as are for example the relations which exist among the phase space degrees of freedom $q^{n}$ and $p_{n}$ independently of $t$ but dependent on the initial values $q_{i}^{n}$ and $p_{n, i}$, such as for instance the energy $E\left(q_{i}^{n}, p_{n, i}\right)=\Lambda$.

The fact that we are indeed dealing with a theory of one-dimensional gravity coupled to matter dynamics may also be justified from another viewpoint. Applying the Hamiltonian reduction of the conjugate momenta $p_{n}$ discussed in Sec. 2 but based this time on the coupled action (15) and the associated equations of motion (17), one readily concludes that the Lagrangian formulation of the coupled system is,

$S\left[q^{n} ; \lambda\right]=\int d \tau\left[L\left(q^{n}, \frac{d q^{n}}{d \tau}\right)+\Lambda\right]=\int d t \lambda(t)\left[L\left(q^{n}, \frac{1}{\lambda(t)} \frac{d q^{n}}{d t}\right)+\Lambda\right]$.

In this form, it is clear that we are indeed dealing with an intrinsic worldline metric defined by the invariant line element

$$
d s^{2}=d t^{2} \lambda^{2}(t)=d \tau^{2},
$$

with $\lambda(t)$ and $\lambda^{2}(t)$ as einbein and metric structures, respectively, coupled in a reparametrisation invariant way to the matter Lagrangian as well as to a world-line cosmological term $\Lambda$. 
Finally, let us consider the local gauge invariance properties in the Hamiltonian formulation. Since $\phi=H-\Lambda$ is the generator for the associated infinitesimal transformations in phase space, their explicit expression reads

$$
\delta_{\epsilon} q^{n}=\epsilon\left\{q^{n}, \phi\right\}=\epsilon \frac{\partial H}{\partial p_{n}} \quad, \quad \delta_{\epsilon} p_{n}=\epsilon\left\{p^{n}, \phi\right\}=-\epsilon \frac{\partial H}{\partial q^{n}} \quad, \quad \delta_{\epsilon} \lambda=\dot{\epsilon},
$$

where $\epsilon(t)$ is some arbitrary function. Indeed, the variation of the first-order action (15) is then a surface term,

$$
\delta_{\epsilon} S\left[q^{n}, p_{n} ; \lambda\right]=\int d t \frac{d}{d t}\left[\epsilon\left(p_{n} \frac{\partial H}{\partial p_{n}}-H+\Lambda\right)\right],
$$

thus expressing the fact that the transformations (25) do define a symmetry of the system, namely world-line reparametrisation invariance of a general matter coupled one-dimensional gravity system.

The abstract canonical quantisation of the system is straightforward enough. The gravitational sector being dynamics free, the space of quantum states is that of the matter sector, as described in Sec. 2, whereas no further quantum operators are associated to the gravitational sector. In particular, the arbitrary Lagrange multiplier function $\lambda(t)$ is not related to a quantum operator, and still serves the sole purpose of parametrising the gauge freedom related to the choice of world-line parametrisation. Compared to the uncoupled quantum matter system, the only modification is that time dependence of states is generated through a Schrödinger equation which now reads

$$
i \hbar \frac{d}{d t}|\psi, t>=\lambda(t)[\hat{H}-\Lambda]| \psi, t>,
$$

or equivalently, in terms of the function $\tau(t)$,

$$
i \hbar \frac{d}{d \tau}|\psi, t(\tau)>=[\hat{H}-\Lambda]| \psi, t(\tau)>,
$$

thus making manifest yet again the fact that $\lambda(t)$ indeed parametrises the freedom in world-line parametrisations.

Given an arbitrary initial state $\left|\psi, t_{i}>=\sum_{n, \alpha_{n}}\right| E_{n}, \alpha_{n}>\psi_{n, \alpha_{n}}$, the solution to the Schrödinger equation is thus now

$$
\left|\psi, t>=\sum_{n, \alpha_{n}}\right| E_{n}, \alpha_{n}>e^{-i / \hbar\left(E_{n}-\Lambda\right) \int_{t_{i}}^{t} d t^{\prime} \lambda\left(t^{\prime}\right)} \psi_{n, \alpha_{n}} .
$$

More generally, the quantum time evolution operator of the quantised system is

$$
\hat{U}\left(t_{2}, t_{1}\right)=\sum_{n, \alpha_{n}}\left|E_{n}, \alpha_{n}>e^{-i / \hbar\left(E_{n}-\Lambda\right)} \int_{t_{1}}^{t_{2}} d t \lambda(t)<E_{n}, \alpha_{n}\right| .
$$


However, all quantum states of the system may not be regarded as being physical, i.e., gauge invariants states, but only those ${ }^{18}$ that are annihilated by the gauge generator $\hat{\phi}=\hat{H}-\Lambda$,

$$
[\hat{H}-\Lambda] \mid \psi_{\text {phys }}>=0 .
$$

When combined with the Schrödinger equation, note that this restriction implies that physical states are independent of time, namely they are indeed independent of the world-line parametrisation as it should.

On the other hand, we also recover for quantum physical states a situation identical to that for the classical gauge invariant solutions, namely the fact that in order for the physical content of the system not to be void, the cosmological constant $\Lambda$ must belong to the $\hat{H}$-spectrum of the matter sector, namely

$$
\Lambda=E_{n_{0}},
$$

for some specific positive integer value of $n_{0}$. In other words, onedimensional quantum gravity coupled to interacting quantum matter is physical provided only the cosmological constant is quantised with a value belonging to the energy spectrum of the quantum matter sector. Clearly, this result is very general, whatever the quantum matter sector. If the latter possesses only a discrete spectrum, $\Lambda$ is quantised within that discrete spectrum, and likewise for a continuous domain in the $\hat{H}$-spectrum.

Let us hence assume that the value $\Lambda$ coincides with one of the energy eigenvalues $E_{n_{0}}$. Consequently, the subspace of physical quantum states is spanned by the states $\mid E_{n_{0}}, \alpha_{n_{0}}>$ with a degeneracy $d_{n_{0}}$,

$$
\left|\psi_{\text {phys }}>=\sum_{\alpha_{n_{0}}}\right| E_{n_{0}}, \alpha_{n_{0}}>\psi_{\alpha_{n_{0}}}, \quad \psi_{\alpha_{n_{0}}}=<E_{n_{0}}, \alpha_{n_{0}} \mid \psi_{\text {phys }}>,
$$

to which the following physical projector ${ }^{11,12}$ is thus associated,

$$
\boldsymbol{E}=\sum_{\alpha_{n_{0}}}\left|E_{n_{0}}, \alpha_{n_{0}}><E_{n_{0}}, \alpha_{n_{0}}\right|, \quad \boldsymbol{E}^{2}=\boldsymbol{E}, \quad \boldsymbol{E}^{\dagger}=\boldsymbol{E} .
$$

In particular, the physical time evolution operator on the physical subspace simply reduces to

$$
\hat{U}_{\text {phys }}\left(t_{2}, t_{1}\right)=\boldsymbol{E} \hat{U}\left(t_{2}, t_{1}\right) \boldsymbol{E}=\sum_{\alpha_{n_{0}}}\left|E_{n_{0}}, \alpha_{n_{0}}><E_{n_{0}}, \alpha_{n_{0}}\right|=\boldsymbol{E} .
$$

Once again, this result expresses the world-line reparametrisation gauge invariance fact that quantum physical states are time independent, as befits any physical state of a quantum theory for gravity. Furthermore, it 
illustrates the general feature that for reparametrisation invariant quantum theories, the physical projector embodies all the physical content of matter coupled quantum gravity. In one dimension, the physics of any such system lies within the subspace of quantum matter states for which the energy coincides with the cosmological constant. It certainly is a fascinating issue to determine how this conclusion extends to higher dimensional matter coupled quantum gravity theories.

The above characterization of the quantum physical subspace is also reminiscent of topological quantum field theories, ${ }^{2,3}$ namely theories of which the reparametrisation invariant physical content is solely dependent on the topology of the underlying manifold, with in particular a finite dimensional space of quantum states. One could debate whether the matter coupled quantum gravity systems of this section define the simplest examples of topological quantum field theories, since the considered world-line topology remains trivial, but they certainly provide the simplest examples of quantum gravity systems with a space of quantum physical states which is finite dimensional and such that the cosmological constant is necessarily quantised in a manner dependent on the quantum matter dynamics to which gravity is coupled, even though the gravitational sector is totally trivial.

\section{Faddeev's Reduced Phase Space Formulation}

Given that the above quantisation of one-dimensional matter coupled quantum gravity is straightforward and free of any ambiguity, including the complete characterization of its physical sector and of its quantum dynamics through the physical projector, it is interesting to confront these results with those that follow from alternative approaches to the quantisation of constrained dynamics, which all rely on some method to fix the gauge freedom associated to first-class constraints. Often, such gauge fixing procedures run into Gribov problems ${ }^{6}$ of one type or another, ${ }^{8}$ rendering the physical interpretation difficult since the quantised system is then no longer physically equivalent to the one originally considered. In contradistinction, the previous approach solely based on the physical projector but not on any gauge fixing procedure whatsoever, is guaranteed to be free of any Gribov ambiguity, ${ }^{13}$ and thus to truly represent the actual quantum dynamics of the original system.

This section considers the application to the previous systems of a quite popular gauge fixing procedure, namely Faddeev's reduced phase space 
approach. ${ }^{4,5}$ This shall be done explicitly for the spherical harmonic oscillator in $N$ dimensions, defined by

$$
H(\vec{q}, \vec{p})=\frac{1}{2 m} \vec{p}^{2}+\frac{1}{2} m \omega^{2} \vec{q}^{2},
$$

but once again, the conclusions will be seen to remain valid in general. Two distinct gauge fixing conditions will be considered, both leading to the conclusion that for this general class of systems, Faddeev's approach is unable to provide their physically correct quantisation as described in Sec. 3 .

The reason why this specific choice of matter sector is made, is that the infinitesimal Hamiltonian world-line reparametrisations (25) may then readily be extended to finite transformations, given by

$$
\begin{aligned}
& \vec{q}^{\prime}(t)=\vec{q}(t) \cos \omega \epsilon(t)+\frac{\vec{p}(t)}{m \omega} \sin \omega \epsilon(t), \\
& \vec{p}^{\prime}(t)=-m \omega \vec{q}(t) \sin \omega \epsilon(t)+\vec{p}(t) \cos \omega \epsilon(t), \\
& \lambda^{\prime}(t)=\lambda(t)+\dot{\epsilon}(t),
\end{aligned}
$$

$\epsilon(t)$ being an arbitrary function, possibly subjected to boundary conditions depending on the choice of boundary conditions on the phase space variables $\vec{q}(t)$ and $\vec{p}(t)$. A straightforward substitution into the first-order action $S[\vec{q}, \vec{p} ; \lambda]=\int d t[\dot{\vec{q}} \cdot \vec{p}-\lambda(H-\Lambda)]$ then finds

$$
\begin{aligned}
& S\left[\vec{q}^{\prime}, \vec{p}^{\prime} ; \lambda^{\prime}\right]=S[\vec{q}, \vec{p} ; \lambda]+ \\
& +\int d t \frac{d}{d t}\left[-\vec{q} \cdot \vec{p} \sin ^{2} \omega \epsilon+\left(\frac{\vec{p}^{2}}{2 m \omega}-\frac{1}{2} m \omega \vec{q}^{2}\right) \sin \omega \epsilon \cos \omega \epsilon+\Lambda \epsilon\right],
\end{aligned}
$$

clearly displaying the gauge invariance of the system under local world-line reparametrisations in phase space. Note that for the present system, these transformations coincide with rotations among the configuration space variables $\vec{q}$ and their conjugate momenta $\vec{p}$, a property related to the fact that energy conservation restricts the dynamics onto an invariant $N$-dimensional torus in phase space.

The latter remark also shows that when this matter system is coupled to one-dimensional gravity, through ${ }^{\mathrm{f}}$

$$
H_{T}=\lambda(t)\left[\frac{1}{2 m} \vec{p}^{2}+\frac{1}{2} m \omega^{2} \vec{q}^{2}-\Lambda\right] \quad, \quad \Lambda>0,
$$

${ }_{\mathrm{f}}$ In this discussion, we take $\Lambda$ to be strictly positive, $\Lambda>0$. A vanishing cosmological constant, $\Lambda=0$, is possible only for the trivial configuration $\vec{q}(t)=\overrightarrow{0}, \vec{p}(t)=\overrightarrow{0}$, while no solutions exist for a negative value of $\Lambda$. 
whatever the configuration solving the equations of motion, there always exists a finite gauge transformation $\epsilon(t)$ such that one of the position (or momentum) degrees of freedom vanishes at all times, say $q^{1}(t)=0$. This property thus suggests to consider a Faddeev reduced phase space formulation of the system defined for instance by the gauge fixing condition ${ }^{12}$

$$
\Omega=q^{1}=0 .
$$

One of the purposes of such a gauge fixing condition is to single out a specific Lagrange multiplier function, namely a specific world-line parametrisation, by requiring this condition to be stable under time evolution. Given the present choice as well as arbitrary nonvanishing values for ${ }^{\mathrm{g}} p_{1}(t) \neq 0$, this implies $\lambda(t)=0$, namely a singular world-line parametrisation such that $\tau(t)=t_{i}$ at all times! Proceeding nonetheless, one finds that the reduced system is represented by the degrees of freedom $\left(q^{i}, p_{i}\right)$ with $i=2,3, \cdots, N$ for which the Dirac brackets are still given by the canonical brackets $\left\{q^{i}(t), p_{j}(t)\right\}=\delta_{i j}$, while the constraint $\phi=H-\Lambda=0$ and the gauge fixing condition $\Omega=0$ are solved by

$$
q^{1}=0 \quad, \quad p_{1}= \pm \sqrt{2 m\left[\Lambda-\sum_{i}\left(\frac{p_{i}^{2}}{2 m}+\frac{1}{2} m \omega^{2} q^{i^{2}}\right)\right]} .
$$

Furthermore, given the value $\lambda(t)=0$, the effective Hamiltonian which generates time evolution on reduced phase space vanishes identically, $H_{\text {red }}=0$.

Consequently, upon canonical quantisation of this reduced phase space formulation of the system, it is clear that no quantisation condition whatsoever on the cosmological constant $\Lambda$ may possibly arise, while at the same time the quantum space of states cannot coincide with the finite dimensional subspace of quantum states of the $N$-dimensional harmonic oscillator for which the energy coincides with a quantised value for the cosmological constant $\Lambda=E_{n_{0}}=\hbar \omega\left(n_{0}+N / 2\right)$, namely specifically ${ }^{12}$ the totally symmetric $S U(N)$ representation of dimension $d_{n_{0}}=\left(N+n_{0}-1\right) ! /\left(n_{0} !(N-1) !\right)$, as established in general terms in Sec. 3. In other words, Faddeev's reduced phase space quantisation of the system associated to the gauge fixing condition $q^{1}=0$ leads to a system of which the physics is totally different

gThe solution $\lambda(t)=0$ may be avoided only if $p_{1}(t)=0$ at all times, which in general is inconsistent with an arbitrary choice of initial values at $t=t_{i}$. It is only if both $q_{i}^{1}=0$ as well as $p_{1, i}=0$ that the $n=1$ degree of freedom decouples altogether from the dynamics. 
from that of the original reparametrisation invariant system, ${ }^{\mathrm{h}}$ a fact which applies in general whenever a given gauge fixing procedure suffers Gribov problems. $^{8}$

Thinking that this conclusion could possibly be related to the singular world-line parametrisation such that $\lambda(t)=0$ which is associated to the choice $q^{1}=0$, one may consider yet another gauge fixing condition, based on the classical solutions to the Hamiltonian equations of motion. Given initial values $\vec{q}_{i}=\vec{q}\left(t_{i}\right)$ and $\vec{p}_{i}=\vec{p}\left(t_{i}\right)$, the general solution is

$$
\begin{aligned}
& \vec{q}(t)=\vec{q}_{i} \cos \omega\left(t-t_{i}\right)+\frac{1}{m \omega} \vec{p}_{i} \sin \omega\left(t-t_{i}\right), \\
& \vec{p}(t)=\vec{p}_{i} \cos \omega\left(t-t_{i}\right)-m \omega \vec{q}_{i} \sin \omega\left(t-t_{i}\right) .
\end{aligned}
$$

For the gravity coupled system, let us thus consider the gauge fixing condition

$$
\Omega=q^{1}-\left[q_{i}^{1} \cos \omega\left(t-t_{i}\right)+\frac{p_{1, i}}{m \omega} \sin \omega\left(t-t_{i}\right)\right] .
$$

Correspondingly, the world-line parametrisation is characterised by the Lagrange multiplier

$$
\lambda(t)=\frac{1}{p_{1}(t)}\left[p_{1, i} \cos \omega\left(t-t_{i}\right)-m \omega q_{i}^{1} \sin \omega\left(t-t_{i}\right)\right],
$$

while the reduced phase space variables are the coordinates $\left(q^{i}, p_{i}\right)$ with $i=2,3, \cdots, N$ for which the Dirac brackets remain canonical, ${ }^{\mathrm{i}}$ with the following solutions to the first-class constraint $\phi=0$ and gauge fixing condition $\Omega=0$,

$$
\begin{aligned}
& q^{1}(t)=q_{i}^{1} \cos \omega\left(t-t_{i}\right)+\frac{p_{1, i}}{m \omega} \sin \omega\left(t-t_{i}\right), \\
& p_{1}(t)= \pm \sqrt{2 m\left[\Lambda-\sum_{i} \frac{p_{i}^{2}}{2 m}-\frac{1}{2} m \omega^{2} \vec{q}^{2}\right]} .
\end{aligned}
$$

Clearly, even though this gauge fixing is nonsingular given the world-line parametrisation with $\tau(t)=t_{i}+\int_{t_{i}}^{t} d t^{\prime} \lambda\left(t^{\prime}\right)$, canonical quantisation of this formulation of the system cannot recover either its correct quantum physical content as established in Sec. 3 with in particular the requirement of a quantised cosmological constant $\Lambda$. Once again, ${ }^{7,8}$ Faddeev's reduced

\footnotetext{
${ }^{\mathrm{h}}$ In fact, following arguments similar to those developed in Ref. 8 but which shall not be pursued here, the reason for this lack of equivalence may be traced back ${ }^{12}$ to Gribov problems of the first and second types associated to the gauge fixing condition $q^{1}=0$.

${ }^{i}$ The reduced phase space Hamiltonian is not given here.
} 
phase space approach fails for these general classes of one-dimensional reparametrisation invariant systems. We are forced to such a conclusion for both examples of gauge fixing conditions above, even though the FaddeevPopov determinant $\{\Omega, \phi\}=p_{1} / m$ does not vanish in either case. Contrary to what is often claimed, though necessary, a nonvanishing Faddeev-Popov determinant is not a sufficient condition for an admissible gauge fixing free of Gribov ambiguities.

It would also be possible to consider these difficulties of Faddeev's reduced phase space approach from the path integral viewpoint, but this issue shall be left aside in this note.

\section{Hamiltonian BRST Quantisation}

Another quite popular approach to constrained dynamics through gauge fixing is the BRST invariant one, namely the BFV-BRST Hamiltonian formalism ${ }^{10}$ appropriate to the canonical quantisation path taken in this note. ${ }^{5}$ Here for simplicity, this quantisation procedure is applied to the onedimensional harmonic oscillator, whose quantisation is best represented in Fock space through creation and annihilation operators $a$ and $a^{\dagger}$ such that $\left[a, a^{\dagger}\right]=\mathbb{1}$ and whose quantum Hamiltonian is

$$
\hat{H}=\hbar \omega\left[a^{\dagger} a+\frac{1}{2}\right] .
$$

Correspondingly, the orthonormalised Fock basis is spanned by the vectors $\left|n>=\left(a^{\dagger}\right)^{n}\right| 0>/ \sqrt{n !}$ with $\langle n| m>=\delta_{n m}$, which are $\hat{H}$-eigenstates, $\hat{H}\left|n>=E_{n}\right| n>$, with $E_{n}=\hbar \omega(n+1 / 2),(n=0,1,2, \cdots)$.

In the Hamiltonian BFV-BRST approach for the gravity coupled system, phase space is extended by introducing a canonical momentum $p_{\lambda}$ conjugate to the Lagrange multiplier $\lambda$, with bracket $\left\{\lambda, p_{\lambda}\right\}=1$, as well as canonical conjugate pairs of ghost degrees of freedom $\eta^{a}$ and $\mathcal{P}_{a}$ of Grassmann odd parity associated to the first-class constraints $G_{a}=\left(p_{\lambda}, \phi\right)$ $(a=1,2)$ with brackets $\left\{\eta^{a}, \mathcal{P}_{b}\right\}=-\delta_{b}^{a}$. Local gauge invariance is then traded for global BRST invariance generated by the nilpotent BRST charge which for the present system reads

$$
Q_{B}=\eta^{1} p_{\lambda}+\eta^{2}\left[\frac{p^{2}}{2 m}+\frac{1}{2} m \omega^{2} q^{2}-\Lambda\right] \quad, \quad\left\{Q_{B}, Q_{B}\right\}=0 .
$$

The ghosts $\eta^{a}$ carry ghost number $(+1)$ and are real under complex conjugation, whereas the ghosts $\mathcal{P}_{a}$ carry ghost number $(-1)$ and are pure imaginary under complex conjugation. 
Time evolution in this extended phase space is generated by a BRST invariant extension of the gauge invariant first-class Hamiltonian of the system. In our case, the general BRST invariant Hamiltonian is of the form

$$
H_{\text {eff }}=-\left\{\Psi, Q_{B}\right\},
$$

where $\Psi$ is some a priori arbitrary function defined over extended phase space, which is pure imaginary under complex conjugation, is of ghost number $(-1)$, and is of odd Grassmann parity. As a matter of fact, gauge fixing of the system is performed through some choice for this function $\Psi$.

Taking for instance

$$
\Psi=F(\lambda) \mathcal{P}_{1}+\lambda \mathcal{P}_{2},
$$

where $F(\lambda)$ is some function of the Lagrange multiplier, leads to the BRST invariant Hamiltonian

$$
H_{\mathrm{eff}}=\lambda\left[\frac{p^{2}}{2 m}+\frac{1}{2} m \omega^{2} q^{2}-\Lambda\right]+F(\lambda) p_{\lambda}-F^{\prime}(\lambda) \mathcal{P}_{1} \eta^{1}-\mathcal{P}_{2} \eta^{1},
$$

and in turn to BRST invariant equations of motion which include

$$
\dot{\lambda}=F(\lambda) \text {. }
$$

Hence, a given choice for the function $F(\lambda)$ and for the initial (or final) value $\lambda_{i}=\lambda\left(t_{i}\right)$ leads to a specific solution $\lambda(t)$, namely a specific worldline parametrisation or gauge fixing of the system.

Choices free of Gribov ambiguities $\operatorname{are}^{7,8} F(\lambda)=\alpha \lambda+\beta$ where $\alpha, \beta$ are arbitrary constant parameters. On the other hand, examples of gauge fixing conditions leading to Gribov problems $\operatorname{are}^{7,8} F(\lambda)=\alpha \lambda^{2}+\beta \lambda+\delta$, $F(\lambda)=\alpha \lambda^{3}$ or $F(\lambda)=e^{-\alpha \lambda}$ with $\alpha \neq 0$. Even though, by construction, the formulation of the system is BRST and thus gauge invariant whatever the choice for $\Psi$ or $F(\lambda)$, the physics that is being described, albeit gauge invariant, does depend on the gauge fixing condition. It is only when the latter is free of any Gribov problem that an admissible gauge fixing has been achieved, and that a description of the system's dynamics which is equivalent to the original one is recovered. The same statement applies already at the classical level ${ }^{8}$ for the solutions to the BRST invariant equations of motion given a choice of BRST invariant boundary conditions. It is only for an admissible choice of gauge fixing function $\Psi$ that the correct classical solutions are recovered, and only those.

By considering the BRST transformations of the extended phase space degrees of freedom, one realises that a general choice of BRST invariant 
boundary conditions, irrespective of the boundary values for the original configuration space coordinates $q^{n}$ at $t=t_{i}$ and $t=t_{f}$, is such that

$$
p_{\lambda}\left(t_{i, f}\right)=0 \quad, \quad \mathcal{P}_{1}\left(t_{i, f}\right)=0, \eta^{2}\left(t_{i, f}\right)=0 .
$$

It is thus also for such a choice of BRST invariant external quantum states that the BRST invariant quantum evolution operator is to be considered, namely that these matrix elements are to represent the gauge invariant time evolution of the quantum system given initial and final $\hat{q}^{n}$-eigenstate configurations $q_{i}^{n}$ and $q_{f}^{n}$, to which only physical states contribute both as intermediate as well as external states.

Canonical quantisation of the BFV-BRST extended system is straightforward. ${ }^{5}$ In the Grassmann even sector, one has the familiar operator algebras $\left[a, a^{\dagger}\right]=\mathbb{1}$ and $\left[\hat{\lambda}, \hat{p}_{\lambda}\right]=i \hbar$. In the ghost sector, one has the fermionic algebra $\left\{\hat{c}^{a}, \hat{b}_{b}\right\}=\delta_{b}^{a}$, with $\hat{c}^{a}=\hat{\eta}^{a}$ and $\hat{b}_{a}=i \hat{\mathcal{P}}_{a} / \hbar$, and the properties $\hat{c}^{a \dagger}=\hat{c}^{a}$ and $\hat{b}_{a}^{\dagger}=\hat{b}_{a}$. The representation of this $(b, c)$ algebra is spanned by a four-dimensional Hilbert space with basis vectors $\mid \pm \pm>$ such that

$$
\begin{array}{lll}
\hat{c}^{1}|-->=|+-> & , & \hat{c}^{1} \mid+->=0, \\
\hat{c}^{1}|-+>=|++> & , & \hat{c}^{1} \mid++>=0, \\
\hat{c}^{2}|-->=|-+> & , & \hat{c}^{2}|+->=-|++>, \\
\hat{c}^{2} \mid-+>=0 & , & \hat{c}^{2} \mid++>=0 \\
\hat{b}_{1} \mid-->=0 & , & \hat{b}_{1} \mid+->=1-->, \\
\hat{b}_{1} \mid-+>=0 & , & \hat{b}_{1} \mid++>=1-+>, \\
\hat{b}_{2} \mid-->=0 & , & \hat{b}_{2} \mid+->=0, \\
\hat{b}_{2}|-+>=|--> & , & \hat{b}_{2}|++>=-|+->,
\end{array}
$$

the inner products of which are defined through the following only nonvanishing matrix elements,

$$
\begin{array}{cc}
<--\mid++>=-\alpha, & <+-\mid-+>=-\alpha, \\
<-+\mid+->=\alpha, & <++\mid-->=\alpha,
\end{array}
$$

with $\alpha= \pm i$. Finally, the antihermitean ghost number operator reads

$$
\hat{Q}_{c}=\frac{1}{2}\left[\hat{c}^{a} \hat{b}_{a}-\hat{b}_{a} \hat{c}^{a}\right],
$$

so that $\mid-->$ is of minimal ghost number $(-1), \mid+->$ and $\mid-+>$ are both of vanishing ghost number, and $\mid++>$ is of maximal ghost number $(+1)$. 
As is characteristic of the BRST construction, physical gauge invariant states correspond to BRST invariant states of minimal ghost number, thus ${ }^{\mathrm{j}}$

$$
\hat{Q}_{B}\left|\psi_{\text {phys }}, t>=0 \quad, \quad \hat{Q}_{c}\right| \psi_{\text {phys }}, t>=-\mid \psi_{\text {phys }}, t>,
$$

where the nilpotent quantum BRST operator is

$$
\hat{Q}_{B}=\hat{c}^{1} \hat{p}_{\lambda}+\hat{c}^{2}\left[\hbar \omega\left(a^{\dagger} a+\frac{1}{2}\right)-\Lambda\right] \quad, \quad \hat{Q}_{B}^{2}=0 .
$$

Consequently once again, in order to obtain a quantum theory with physical content, it is necessary that the cosmological constant $\Lambda$ belongs to the matter energy spectrum, namely in the present case

$$
\Lambda=\hbar \omega\left(n_{0}+\frac{1}{2}\right),
$$

for some positive integer value $n_{0}$, a condition which we shall henceforth assume to be met. The general solution for physical states at minimal ghost number is then of the form

$$
\left|\psi_{\text {phys }}, t>=\right| n_{0}>\otimes\left|p_{\lambda}=0>\otimes\right|-->\psi_{--, n_{0}}(t),
$$

$\psi_{--, n_{0}}(t)$ being an arbitrary complex function of time, which turns out to be a constant when solving the Schrödinger equation hereafter. Here, $\mid p_{\lambda}>$ denotes the $\hat{p}_{\lambda}$-eigenstate basis normalised such that $\left\langle p_{\lambda} \mid p_{\lambda}^{\prime}\right\rangle=\delta\left(p_{\lambda}-p_{\lambda}^{\prime}\right)$.

As a matter of fact, it is possible to solve for all BRST invariant states irrespective of their ghost number, $\hat{Q}_{B} \mid \psi, t>=0$, leading to the following general decomposition,

$$
\begin{aligned}
\mid \psi, t>= & \left|n_{0}>\otimes\right| p_{\lambda}=0>\otimes \mid-->\psi_{--, n_{0}}(t) \\
& +\left|n_{0}>\otimes\right| p_{\lambda}=0>\otimes \mid+->\psi_{+-, n_{0}}(t) \\
& +\left|n_{0}>\otimes\right| p_{\lambda}=0>\otimes \mid-+>\psi_{-+, n_{0}}(t) \\
& +\left|n_{0}>\otimes\right| p_{\lambda}=0>\otimes\left|++>\psi_{++, n_{0}}(t)+\hat{Q}_{B}\right| \chi, t>,
\end{aligned}
$$

where $\mid \chi, t>$ is an arbitrary quantum state. In this form, the BRST cohomology classes are explicit as well, showing that there are four distinct nontrivial BRST invariant cohomology classes, each of dimension unity. Besides the trivial cohomology class which includes states of ghost numbers 0 and $(+1)$, all four nontrivial classes are in one-to-one correspondence with each of the four basis states $\mid \pm \pm>$ in the ghost sector. A priori, each of these nontrivial classes could also be put into one-to-one correspondence

${ }^{\mathrm{j} A s}$ we shall see, upon imposing Schrödinger's equation, physical states are also time independent. 
with the physical states spanned by $\mid n_{0}>$ that define the correct quantisation of this system as constructed in Sec. 3, up to the product with the $\hat{p}_{\lambda}$-eigenstate $\mid p_{\lambda}=0>$. However, when considering the time evolution of BRST invariant states generated by the Schrödinger equation to be discussed below, it turns out that only the BRST nontrivial cohomology class of minimal ghost number $(-1)$ always possesses the correct time dependent dynamics irrespective of the choice of gauge fixing fermion $\Psi$, leading back precisely to the characterization of physical states given in (59) as being only those BRST invariant states that are of minimal ghost number. This is a general feature of Hamiltonian BRST quantisation whatever the gauge invariant system being considered. On the other hand, even though in the present system it might appear that such a one-to-one correspondence with Dirac's identification of physical states could possibly also apply for the other nontrivial cohomology classes at ghost numbers 0 and $(+1)$, whether their correct time evolution is recovered as well is again a matter dependent on the choice of gauge fixing function $\Psi$, and is generally not realised, even for an admissible gauge fixing.

Furthermore, as we shall see presently, the presence of the extended phase space degrees of freedom, leading to the $\left|p_{\lambda}=0>\otimes\right|-->$ component of the physical states, implies an ill defined normalisation of BRST invariant matrix elements of the BRST invariant quantum evolution operator, namely in fine, of the physical states themselves. For instance, although the physical component $\mid n_{0}>$ is properly normalised to unity, that of the BRST invariant physical state (59) itself, whether $\psi_{--, n_{0}}(t)$ is a pure phase factor or not, is ill defined since the inner product of the state $\mid p_{\lambda}=0>$ with itself is the $\delta$-function $\delta(0)$ while that of the state $\mid-->$ with itself vanishes, leading to an undetermination of the form $0 \cdot \delta(0)$ for the inner product of the physical states in (59).

The same issue also arises for BRST invariant matrix elements of the BRST invariant quantum evolution operator of the system. Given the class of gauge fixing functions of the form (49), the corresponding BRST invariant quantum Hamiltonian is

$\hat{H}_{\mathrm{eff}}=\frac{i}{\hbar}\left\{\hat{\Psi}, \hat{Q}_{B}\right\}=\hat{\lambda}\left[\hbar \omega\left(a^{\dagger} a+\frac{1}{2}\right)-\Lambda\right]+F(\hat{\lambda}) \hat{p}_{\lambda}-i \hbar \hat{c}^{1} \hat{b}_{1} F^{\prime}(\hat{\lambda})-i \hbar \hat{c}^{1} \hat{b}_{2}$,

for which the Schrödinger equation over the extended space of quantum states reads

$$
i \hbar \frac{d}{d t}\left|\psi, t>=\hat{H}_{\mathrm{eff}}\right| \psi, t>
$$


Applied to the nontrivial BRST cohomology classes in (60), one finds that for this class of gauge fixing choices only $\psi_{--, n_{0}}(t)$ and $\psi_{-+, n_{0}}(t)$ are necessarily time independent as is required for actual gauge invariant states in Dirac's approach, whereas $\psi_{+-, n_{0}}(t)$ never is, while $\psi_{++, 0}(t)$ would be provided only $F(\lambda)$ were to be constant, which still excludes a large class of admissible gauge fixing choices. Hence, it is only the cohomology class of minimal ghost number constructed in (59) that always also solves the Schrödinger equation in a manner consistent with the actual identification of physical states in Dirac's quantisation, namely in the present case with a time independent component $\psi_{--, n_{0}}$. In spite of the normalisation problems just mentioned, there thus appears a one-to-one correspondence between the physical states $\mid n_{0}>\psi_{--, n_{0}}$ of Dirac's quantisation as discussed in Sec. 3 and the BRST invariant physical states of minimal ghost number constructed here. The physical content should thus be equivalent in both approaches, provided however that time evolution in the BRST approach be also consistent with that in Dirac's approach.

To understand how gauge fixing and Gribov problems may interfere with such an equivalence precisely at that level, let us now consider the BRST invariant quantum evolution operator associated to a given choice of gauge fixing function $\Psi$, namely the operator

$$
\hat{U}_{\mathrm{BRST}}\left(t_{2}, t_{1}\right)=e^{-i / \hbar\left(t_{2}-t_{1}\right) \hat{H}_{\text {eff }}}=e^{\left(t_{2}-t_{1}\right)\left\{\hat{\Psi}, \hat{Q}_{B}\right\} / \hbar^{2}} .
$$

Naively, matrix elements of this operator between BRST invariant states should be independent of the gauge fixing function $\Psi$, since the BRST charge is nilpotent, $\hat{Q}_{B}^{2}=0$. Indeed, given two states $\left|\psi_{1}\right\rangle$ and $\left|\psi_{2}\right\rangle$ that are annihilated by $\hat{Q}_{B}$, it would appear ${ }^{10}$ that through a direct expansion of the exponential, one has simply

$$
<\psi_{2}\left|\hat{U}_{\mathrm{BRST}}\left(t_{2}, t_{1}\right)\right| \psi_{1}>=<\psi_{2} \mid \psi_{1}>
$$

thus apparently establishing that BRST invariant matrix elements of $\hat{U}_{\mathrm{BRST}}\left(t_{2}, t_{1}\right)$ are totally independent of the choice of gauge fixing function $\Psi$, namely the usual statement of the Fradkin-Vilkovisky theorem. ${ }^{10}$ However as we have observed, the matrix element on the r.h.s. of this relation is ill defined for nontrivial BRST cohomology classes (or at best it vanishes identically, which is certainly not what should be expected from matrix elements representing a quantum system with actual physical content), so that the l.h.s. requires further specification as to its explicit evaluation, thereby possibly implying that even though such matrix elements are indeed BRST invariant, and thus gauge invariant as well, they may not be 
totally independent of the gauge fixing function $\Psi$. Indeed, this is what actually happens, and once again, it is only for an admissible gauge fixing, namely such that each of the distinct gauge orbits of the dynamical system is effectively singled out once and only once, that the correct physics is represented through the above matrix elements. ${ }^{7,8}$ Nevertheless in the general case, the above matrix elements do depend on the choice of gauge fixing but actually then only through the gauge equivalence class to which that gauge fixing belongs, these gauge equivalence classes being defined through the covering of the space of gauge orbits which is induced by the gauge fixing. In fact, this is the actual content of the Fradkin-Vilkovisky theorem..$^{7,8}$

As a first illustration of these features within the present example, let us consider the zero ghost number BRST invariant states that are associated to the BRST invariant boundary conditions (52), namely

$$
\begin{aligned}
\mid \psi_{i}> & =\left|q_{i}>\otimes\right| p_{\lambda}=0>\otimes \mid-+> \\
& =\sum_{n=0}^{\infty}|n>\otimes| p_{\lambda}=0>\otimes|-+><n| q_{i}>, \\
\mid \psi_{f}> & =\left|q_{f}>\otimes\right| p_{\lambda}=0>\otimes \mid-+> \\
& =\sum_{n=0}^{\infty}|n>\otimes| p_{\lambda}=0>\otimes|-+><n| q_{f}>,
\end{aligned}
$$

where $\mid q>$ denotes the $\hat{q}$-eigenstate basis normalised such that ${ }^{\mathrm{k}}\langle q| q^{\prime}>=$ $\delta\left(q-q^{\prime}\right)$. However, a direct evaluation of the matrix elements

$$
<n ; p_{\lambda}=0 ;-+\left|\hat{U}_{\mathrm{BRST}}\left(t_{2}, t_{1}\right)\right| m ; p_{\lambda}=0 ;-+>,
$$

leads to an undetermination of the form $0 \cdot \delta(0)$, as explained previously. To avoid this ambiguity, one may momentarily relax the requirement of BRST invariance of the external states ${ }^{1}$ and consider rather the matrix elements

$$
<n ; p_{\lambda, 2} ;-+\left|\hat{U}_{\mathrm{BRST}}\left(t_{2}, t_{1}\right)\right| m ; p_{\lambda, 1} ;-+>\text {. }
$$

Being no longer BRST invariant, this expression may now acquire a well defined value which, however, may also depend on the choice for $\Psi$. Hence even in the limit $p_{\lambda, 1}, p_{\lambda, 2} \rightarrow 0$ of BRST invariant external states, which might lead to a distribution-like expression, it could be that the final result obtained through this form of evaluation through a continuity procedure

${ }^{\mathrm{k}}$ The matrix elements $\langle n \mid q\rangle$ are thus the harmonic oscillator energy eigenstate configuration space wave functions in the case of our specific example.

${ }^{1}$ An alternative approach which maintains explicit BRST invariance at all stages is presented in Ref. 19, confirming once again the discussion developed here. 
of an otherwise ill defined expression retains some dependence on $\Psi$, albeit a gauge invariant one through the induced covering of the space of gauge orbits. As we shall see, this is indeed the case, and as a general result is the actual content of the Fradkin-Vilkovisky theorem..$^{7,8}$

The evaluation of the matrix elements (67) is best performed through a path integral representation. The $\left(\lambda, p_{\lambda}\right)$ and ghost sectors being common to all one-dimensional reparametrisation invariant systems, and thus common to that of the relativistic scalar particle in a Minkowski spacetime, one may borrow the result of the path integral evaluation over these degrees of freedom directly from that latter system. ${ }^{7,8}$ With the following choice of sign for the ghost matrix elements $<--|++>=i=-<-+|+->$, and given the class of gauge fixing functions $\Psi$ in (49), one then finds the exact and explicit result,

$$
\begin{aligned}
\lim _{p_{\lambda, 1}, p_{\lambda, 2} \rightarrow 0}<n ; p_{\lambda, 2} ;-+\mid & \hat{U}_{\mathrm{BRST}}\left(t_{2}, t_{1}\right) \mid m ; p_{\lambda, 1} ;-+>= \\
= & i \delta_{n, m} \int_{\mathcal{D}} \frac{d \gamma}{2 \pi \hbar} e^{-i \gamma / \hbar(\hbar \omega(n+1 / 2)-\Lambda)},
\end{aligned}
$$

where the real Teichmüller parameter ${ }^{7,8}$

$$
\gamma=\int_{t_{1}}^{t_{2}} d t \lambda(t)
$$

the Lagrange multiplier $\lambda(t)$ and the domain of integration $\mathcal{D}$ are constructed as follows. Given the gauge fixing condition

$$
\dot{\lambda}=F(\lambda)
$$

associated to (49), and an arbitrary integration constant $\lambda_{f}=\lambda\left(t_{f}\right)$, consider the corresponding solution $\lambda\left(t ; \lambda_{f}\right)$ to this equation as well as its Teichmüller parameter $\gamma\left(\lambda_{f}\right)$. As the integration constant $\lambda_{f}$ covers the interval $-\infty<\lambda_{f}<+\infty$ once with that orientation, the Teichmüller parameter $\gamma\left(\lambda_{f}\right)$ covers a certain oriented domain $\mathcal{D}$ in the real line. This is the domain over which the final integral in (68) must be performed, including its orientation. This is also how all the extended phase space degrees of freedom contribute to the final matrix element, leading to a dependence on the Lagrange multiplier only through its gauge invariant Teichmüller parameter. $^{\mathrm{m}}$

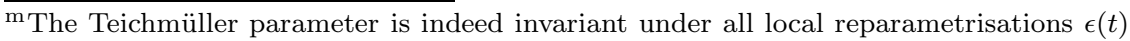
consistent with the chosen boundary conditions at $t=t_{i, f}$ for the matter degrees of freedom, namely $\epsilon\left(t_{i, f}\right)=0$. 
Even though the final expression (68) is indeed manifestly BRST and gauge invariant, it does depend on the choice of gauge fixing function $\Psi$ or $F(\lambda)$ in as far as the domain of integration $\mathcal{D}$ depends on that choice, namely precisely through the covering of the space of gauge orbits that is induced by the choice of gauge fixing function $F(\lambda)$. An admissible gauge fixing is such that the domain $\mathcal{D}$ coincides exactly once with the real line, ${ }^{7,8}$ which is the case for example for $F(\lambda)=\alpha \lambda+\beta$. However, if one considers for instance $F(\lambda)=\alpha \lambda^{3}$ with $\alpha>0$ and $\left(t_{2}-t_{1}\right)>0$, the domain $\mathcal{D}$ is such that the Teichmüller parameter $\gamma$ ranges from $-\sqrt{2\left(t_{2}-t_{1}\right) / \alpha}$ to $\sqrt{2\left(t_{2}-t_{1}\right) / \alpha}$, clearly displaying the dependence of (68) on the parameter $\alpha$ defining the gauge fixing condition, albeit in a gauge invariant manner. Nevertheless, in the limit $\alpha \rightarrow 0$, one recovers the domain associated to an admissible gauge fixing, as is indeed then also the choice $F(\lambda)=0$. Hence, contrary to what is often stated, the BRST invariant matrix elements of the BRST invariant quantum evolution operator are not totally independent of the gauge fixing condition, but depend on it only in as far as the induced covering of the space of gauge orbits is dependent on the choice of gauge fixing. For a gauge fixing procedure suffering Gribov ambiguities, such as the choices $F(\lambda)=\alpha \lambda^{3}$ or $F(\lambda)=\alpha \lambda^{2}+\beta \lambda+\delta$ when $\alpha \neq 0$, one does not represent the same quantum dynamics as that of the original system, in spite of the fact that the description remains manifestly gauge invariant irrespective of the gauge fixing choice and procedure. Gauge invariance is not all there is to gauge invariant systems!

Assuming now an admissible gauge fixing choice to have been made, in which case the integral in (68) is over the entire real line, one thus finally has the following evaluation of the relevant BRST invariant matrix elements which represent the quantum propagator for physical states within the BFV-BRST Hamiltonian formalism,

$<n ; p_{\lambda}=0 ;-+\left|\hat{U}_{\mathrm{BRST}}\left(t_{2}, t_{1}\right)\right| m ; p_{\lambda}=0 ;-+>=i \delta_{n, m} \delta\left(\hbar \omega\left(n+\frac{1}{2}\right)-\Lambda\right)$.

Hence once again, but clearly only provided the gauge fixing procedure is free of any Gribov ambiguity, one recovers the requirement of a quantised value of the cosmological constant which must belong to the energy spectrum of the matter sector, namely in the present instance for some specific positive integer $n_{0}$,

$$
\Lambda=\hbar \omega\left(n_{0}+\frac{1}{2}\right),
$$

in which case the physical subspace is also confined to the states spanned 
by $\mid n_{0}>$ in the matter sector.

As a matter of fact, provided a specific choice for $F(\lambda)$ is made at the outset, it is also possible to circumvent the path integral evaluation of the relevant matrix element. For example given the admissible gauge fixing with $F(\lambda)=0$, as well as the following non-BRST invariant external states

$$
\begin{aligned}
\mid \psi_{i}> & =\int_{-\infty}^{\infty} d p_{\lambda} \mid n ; p_{\lambda} ;-+>\psi_{i}\left(p_{\lambda}\right), \\
\mid \psi_{f}> & =\int_{-\infty}^{\infty} d p_{\lambda} \mid m ; p_{\lambda} ;-+>\psi_{f}\left(p_{\lambda}\right),
\end{aligned}
$$

an explicit calculation of the matrix element $<\psi_{f}\left|\hat{U}_{\mathrm{BRST}}\left(t_{2}, t_{1}\right)\right| \psi_{i}>$ is readily performed, with the result

$$
\begin{aligned}
& <\psi_{f}\left|e^{-i / \hbar\left(t_{2}-t_{1}\right) \hat{H}_{\text {eff }}}\right| \psi_{i}>= \\
& =i \delta_{n, m}\left(t_{2}-t_{1}\right) \int_{-\infty}^{\infty} d \lambda \tilde{\psi}_{f}^{*}(\lambda) e^{-i / \hbar \lambda\left(t_{2}-t_{1}\right)(\hbar \omega(n+1 / 2)-\Lambda)} \tilde{\psi}_{i}(\lambda),
\end{aligned}
$$

where

$$
\tilde{\psi}_{i, f}(\lambda)=\int_{-\infty}^{\infty} \frac{d p_{\lambda}}{\sqrt{2 \pi \hbar}} e^{i / \hbar \lambda p_{\lambda}} \psi_{i, f}\left(p_{\lambda}\right) .
$$

In the limit of BRST invariant external states, namely $\delta$-peaked in the conjugate momentum $p_{\lambda}$ with $\psi_{i, f}\left(p_{\lambda}\right)=\delta\left(p_{\lambda}\right)$ and thus non-normalisable, one recovers the above expression (68) in the case of an admissible gauge fixing condition, namely with $\mathcal{D}$ being the entire real line,

$$
\begin{aligned}
<n ; p_{\lambda}=0 ;-+\mid & \hat{U}_{\mathrm{BRST}}\left(t_{2}, t_{1}\right) \mid m ; p_{\lambda}=0 ;-+>= \\
& =i \delta_{n, m} \int_{-\infty}^{\infty} \frac{d \gamma}{2 \pi \hbar} e^{-i / \hbar \gamma(\hbar \omega(n+1 / 2)-\Lambda)},
\end{aligned}
$$

and thus the same final expression and conclusion as in (71). Nevertheless, one sees that whatever the approach, the evaluation of such BRST invariant matrix elements, associated to the quantum propagator of physical states, requires first an evaluation for non-BRST invariant external states, leading therefore to the possibility of a dependence of gauge invariant quantities on the gauge fixing conditions, albeit in a gauge invariant manner. In fact, it is possible to circumvent the ill defined singular products $0 \cdot \delta(0)$ stemming from the non-normalisable character of the $\hat{p}_{\lambda}$ eigenstates in still another manner, that also explicitly preserves manifest BRST invariance at all stages and does not require to consider $\hat{U}_{\mathrm{BRST}}\left(t_{2}, t_{1}\right)$ matrix elements for non-BRST invariant external states, and still reach exactly the identical conclusion. ${ }^{19}$ Any gauge fixing leads to a dynamics defined over the space of gauge orbits of the system, hence to a gauge invariant dynamics, but 
which of these gauge orbits are included and with which multiplicity is dependent on the choice of gauge fixing procedure. The correct physics and dynamics is represented only for an admissible gauge fixing procedure, namely one which selects, up to a common weight factor, once and only once each of the gauge orbits and thus induces an admissible covering of the space of gauge orbits. And it is only for such an admissible gauge fixing that the correct quantisation of the cosmological constant is reproduced within BRST quantisation.

As a final remark, note that when the cosmological constant takes a quantised valued within the energy spectrum of the matter sector, $\Lambda=E_{n_{0}}$, the relevant BRST invariant matrix elements are given in terms of the $\delta\left(E_{n}-\Lambda\right)$ distribution with a singular value for the physical sector $n=n_{0}$, yet another manifestation of the lack of normalisability of physical states within the BRST quantisation approach which is due to the extended phase space degrees of freedom. This situation is to be contrasted with the identification of physical states within Dirac's approach, in which everything is so much more straightforward and transparent when expressed in terms of the physical projector, including the dynamical time evolution of physical quantum states.

\section{Conclusions}

Inspired by a simple example discussed in Ref. 12, this note has discussed the coupling of quantum gravity in one (time) dimension to an arbitrary quantum mechanical interacting matter sector. One of the main conclusions of this analysis is that, as a very general result, and even though onedimensional gravity is quantum dynamics free, the cosmological constant in such systems needs to take a value that belongs to the energy spectrum of the quantum matter sector, and thus to be quantised, while physical states are then also confined to the subspace of the matter quantum states for which the energy coincides with the cosmological constant value. These are certainly very intriguing conclusions, which call for their possible extension to higher dimensional theories of quantum gravity coupled to interacting quantum matter, hopefully eventually shedding some new light onto the cosmological constant problem.

The quantisation of these one-dimensional matter coupled quantum gravity systems is most efficient and simple based on the physical projector approach, ${ }^{11,12}$ lending itself to the immediate identification of the gauge in- 
variant physical sector and its quantum dynamics, and of the quantisation requirement on the cosmological constant. Such a circumstance enables one to contrast the efficacy of that approach to that of other methods developed over the years towards the quantisation of gauge invariant, and more generally, of constrained dynamics.

As has been explicitly illustrated, Faddeev's reduced phase space approach ${ }^{4}$ simply fails for one-dimensional reparametrisation invariant systems, for reasons that may be traced back to the appearance of Gribov ambiguities whatever the gauge fixing conditions that might be introduced..$^{8,12}$ Even though the resulting formulation remains gauge invariant, it is not independent of the gauge fixing conditions, ${ }^{8}$ and thus does not necessarily reproduce the same quantum physics as that of the original system, as is indeed always the case in the presence of Gribov problems. Given that most approaches towards quantum gravity rely one way or another on Faddeev's gauge fixing procedure, the fact that it already fails in one dimension because of Gribov problems and thus cannot capture the then necessary quantisation condition on the cosmological constant leaves totally open the possibility that such a quantisation is also required in higher dimensions.

The Hamiltonian BRST invariant quantisation method ${ }^{10}$ has also been applied to these classes of systems, to explicitly illustrate once again the dependence of the resulting quantum dynamics on the gauge fixing condition. ${ }^{7,8}$ It is only for an admissible gauge fixing procedure that BRST invariant physical states as well as the actual BRST invariant quantum dynamics are in one-to-one correspondence with the physical states and their correct quantum dynamics as readily identified within Dirac's approach through the physical projector, and that the requirement of a quantised cosmological constant is recovered. Nevertheless, the introduction of extended degrees of freedom, among which the ghost sector required to balance the gauge variant variables, implies that BRST invariant physical states correspond to non-normalisable quantum states, a technical difficulty which is avoided altogether within Dirac's approach and the physical projector.

These general classes of one-dimensional matter coupled quantum gravity models clearly illustrate the various advantages of the physical projector approach, in which no gauge fixing procedure whatsoever is required and which thus from the outset avoids ${ }^{13}$ having to address the difficult and subtle issues of Gribov problems which plague essentially any gauge fixing conditions being used for systems of physical interest. Beginning with the intriguing question regarding the possible quantised value of the cosmo- 
logical constant and the restriction of physical states to the subspace of matter quantum states whose energy coincides with cosmological constant value, the exploration of the efficacy and the new insights that the physical projector may bring to constrained dynamics thus appears to be of potential great interest to the understanding of the gauge invariant theories on which our present description of the physical world and its fundamental interactions is based. ${ }^{14,20}$ Such an understanding might also bring us closer to determining whether the suggestion made in the opening paragraph of the Introduction has any chance of being physically meaningful.

\section{Acknowledgements}

The results of this work have been available since February 2002 as a STIAS preprint. ${ }^{21}$ It is my great pleasure to dedicate it to John R. Klauder on the occasion of his $70^{\text {th }}$ birthday, as a tribute to his many scientific insights and his warm friendship. The author also wishes to thank the Stellenbosch Institute for Advanced Study (STIAS) and its Director, Prof. Bernard Lategan, for financial support making possible my participation in the STIAS hosted Workshop on String Theory and Quantum Gravity (February 4-22, 2002), a most enjoyable and rewarding experience indeed, during which this work was completed. My thanks also go out to Prof. Hendrik Geyer, organiser of the Workshop, and Prof. Frederik Scholtz, Head of the Department of Physics at the University of Stellenbosch, for their wonderful hospitality, and for fruitful scientific discussions. This work is partially supported by the Federal Office for Scientific, Technical and Cultural Affairs (Belgium) through the Interuniversity Attraction Pole P5/27.

\section{References}

1. For reviews on the cosmological constant problem, see for instance, S. Weinberg, Rev. Mod. Phys. 61, 1 (1989); The Cosmological Constant Problems, e-print arXiv:astro-ph/0005265 (May 2000);

E. Witten, The Cosmological Constant from the Viewpoint of String Theory, e-print arXiv:hep-ph/0002297 (February 2000).

2. E. Witten, Comm. Math. Phys. 117, 353 (1988);

E. Witten, Comm. Math. Phys. 121, 351 (1988).

3. For a review, see,

D. Birmingham, M. Blau, M. Rakowski and G. Thompson, Physics Reports 209, 129 (1991).

4. L.D. Faddeev, Theor. Math. Phys. 1, 1 (1970). 
5. For a detailed discussion and references to the original literature, see for example Ref. 8. An introduction to the subject of constrained quantisation is also available from Ref. 9.

6. V.N. Gribov, Nucl. Phys. B139, 1 (1978); I.M. Singer, Comm. Math. Phys. 60, 7 (1978).

7. J. Govaerts, Int. J. Mod. Phys. A4, 173 (1989);

J. Govaerts, Int. J. Mod. Phys. A4, 4487 (1989);

J. Govaerts and W. Troost, Class. Quantum Grav. 8, 1723 (1991).

8. J. Govaerts, Hamiltonian Quantisation and Constrained Dynamics (Leuven University Press, Leuven, 1991).

9. J. Govaerts, The quantum geometer's universe: particles, interactions and topology, in the Proceedings of the Second International Workshop on Contemporary Problems in Mathematical Physics, J. Govaerts, M.N. Hounkonnou and A.Z. Msezane, eds. (World Scientific, Singapore, 2002), pp. 79-212.

10. E.S. Fradkin and G.A. Vilkovisky, Phys. Lett. B55, 224 (1975);

I.A. Batalin and G.A. Vilkovisky, Phys. Lett. B69, 309 (1977);

E.S. Fradkin and T.E. Fradkina, Phys. Lett. B72, 343 (1978);

I.A. Batalin and E.S. Fradkin, Rivista Nuovo Cimento 9, 1 (1986).

11. J.R. Klauder, Ann. Phys. 254, 419 (1997);

J.R. Klauder, Nucl. Phys. B547, 397 (1999).

12. J.R. Klauder, Quantization of Constrained Systems, Lect. Notes Phys. 572, 143 (2001), e-print arXiv:hep-th/0003297 (March 2000).

13. J. Govaerts, J. Phys. A30, 603 (1997).

14. J.R. Klauder, J. Math. Phys. 40, 5860 (1999);

G. Watson and J.R. Klauder, J. Math. Phys. 41, 8072 (2000);

J.R. Klauder, J. Math. Phys. 42, 4440 (2001);

J.R. Klauder, The Affine Quantum Gravity Program, Class. Quant. Grav. 19, 817 (2002), e-print arXiv:gr-qc/0110098 (October 2001);

G. Watson and J.R. Klauder, Metric and Curvature in Gravitational Phase Space, Class. Quant. Grav. 19, 3617 (2002), e-print arXiv:gr-qc/0112053 (December 2001).

15. K. Fujikawa, Prog. Theor. Phys. 96, 863 (1996);

K. Fujikawa, Nucl. Phys. B484, 495 (1997).

16. P.F. González-Díaz, Mod. Phys. Lett. A2, 551 (1987);

I. Moss, Phys. Lett. B283, 52 (1992);

R. Bousso and J. Polchinski, JHEP 0006, 006 (2000).

17. A quantisation condition on the cosmological constant has also been obtained within the loop quantum gravity programme for quantum gravity; see for instance,

L. Smolin, J. Math. Phys. 36, 6417 (1995);

S. Major and L. Smolin, Nucl. Phys. B473, 267 (1996);

R. Borissov, S. Major and L. Smolin, Class. Quant. Grav. 13, 3183 (1996);

R. Gambini and J. Pullin, Phys. Rev. Lett. 85, 5272 (2000); Class. Quant. Grav. 17, 4515 (2000).

For a recent review on loop quantum gravity, see,

A. Ashtekar and J. Lewandowski, Class. Quant. Grav. 21, R53 (2004). 
18. P.A.M. Dirac, Lectures on Quantum Mechanics (New York, Belfer Graduate School of Science, Yeshiva University, 1964).

19. J. Govaerts and F.G. Scholtz, J. Phys. A37, 7359 (2004).

20. J. Govaerts and J.R. Klauder, Ann. Phys. 274, 251 (1999); V.M. Villanueva, J. Govaerts and J.-L. Lucio-Martinez, J. Phys. A33, 4183 (2000);

J. Govaerts and B. Deschepper, J. Phys. A33, 1031 (2000).

21. J. Govaerts, The Cosmological Constant of One-Dimensional Matter Coupled Quantum Gravity is Quantized, preprint STIAS-02-002, e-print arXiv: hep-th/0202134 (February 2002). 